\title{
DÜBLIN
}

Technological University Dublin

ARROW@TU Dublin

Articles

School of Electrical and Electronic Engineering

2011-08-02

\section{A Microfiber Cavity with Minimal-Volume Confinement}

Ming Ding

University of Southampton

Pengfei Wang

Technological University Dublin, pengfei.wang@tudublin.ie

Timothy Lee

University of Southampton

See next page for additional authors

Follow this and additional works at: https://arrow.tudublin.ie/engscheceart

Part of the Electromagnetics and Photonics Commons

\section{Recommended Citation}

Ding, M., Wang, P., Lee, T., Brambilla, G.:A Microfiber Cavity with Minimal-Volume Confinement. Applied Physics Letters, Vol. 99, 5, 2011, article no. 051105. doi:10.1063/1.3621836

This Article is brought to you for free and open access by the School of Electrical and Electronic Engineering at ARROW@TU Dublin. It has been accepted for inclusion in Articles by an authorized administrator of ARROW@TU Dublin. For more information, please contact arrow.admin@tudublin.ie, aisling.coyne@tudublin.ie, gerard.connolly@tudublin.ie.

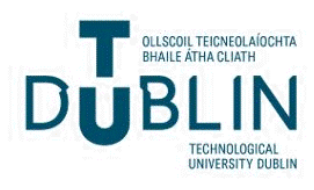




\section{Authors}

Ming Ding, Pengfei Wang, Timothy Lee, and Gilberto Brambilla 


\title{
A microfiber cavity with minimal-volume confinement
}

\author{
Ming Ding, ${ }^{\text {a) }}$ Pengfei Wang, Timothy Lee, and Gilberto Brambilla \\ Optoelectronics Research Centre, University of Southampton, Southampton, SO17 IBJ, United Kingdom
}

(Received 7 June 2011; accepted 11 July 2011; published online 2 August 2011)

\begin{abstract}
We demonstrate a type of microcavity with minimal-volume confinement using a high-contrast phase-shifted Bragg grating in a microfiber. While waveguiding by the air-silica boundary provides a diffraction-limited two-dimensional confinement, the grating introduces the third degree of confinement. Theoretical simulations verified the microfiber cavity confinement while the experimental demonstration, carried out in samples nanostructured by focused ion beam, showed a good agreement with theoretical predictions. This cavity can be used for a variety of applications ranging from sensing to quantum dynamic experiments. () 2011 American Institute of Physics. [doi:10.1063/1.3621836]
\end{abstract}

In recent years, resonators based on optical microcavities have attracted increasing attention ${ }^{1}$ because of their wide range of applications. These objects can confine light in a small volume by resonant recirculation and have been demonstrated in several structures. ${ }^{1}$ Whispering gallery mode obtained extreme light confinement within volumes of $\sim 10^{3}$ $10^{5} \mu \mathrm{m}^{3}$ and have been observed in several geometries including microtoroid, ${ }^{2}$ microsphere, ${ }^{3}$ and microdisk $^{4}$ resonators. Microcavities based on photonic crystals can also provide extremely small mode volumes $\left(<1 \mu \mathrm{m}^{3}\right):^{5}$ in 1999 , a photonic crystal in which one hole was left unetched allowed for the creation of an optical microcavity where a half wavelength thick waveguide allowed for vertical confinement, while forming a photonic crystal mirror provided a two-dimensional lateral localization.

Another type of resonator used Fabry-Perot cavities manufactured in a quantum-dot-loaded micropillar: ${ }^{6}$ in this device, Bragg mirrors provide longitudinal confinement, whereas air-dielectric guiding provides radial confinement. Fabry-Perot microcavities have also been demonstrated in optical fibers, ${ }^{7}$ but the volume was in the order of $10^{3}-10^{4}$ $\mathrm{mm}^{3}$ because of the small refractive index contrast $\left(10^{-3}\right)$ in the grating fringes and at the core/cladding interface.

In this paper, a microfiber phase-shifted Bragg grating (PSBG) cavity is proposed for three-dimensional light confinement. Since in optical microfibers light is radially confined by the cladding/air interface, for appropriate taper diameters the diffraction limit can be achieved. Longitudinally, confinement is provided by the phase shift in the center of the PSBG: the distance along which confinement occurs is related to the refractive index contrast of the grating. In order to maximize such confinement (thus minimize confinement length), PSBGs were manufactured carving notches in the microfiber, thus achieving an index contrast of $\sim 0.45$ (difference between silica refractive index $\mathrm{n}_{\text {Silica }} \sim 1.45$ and air refractive index $\mathrm{n}_{\text {Air }} \sim 1$ ) at the wavelength $\lambda \sim 1 \mu \mathrm{m}$.

In optical fibers the small refractive index difference in the grating pitches implies that thousands of periods are needed to provide strong reflectivity; on the contrary, in

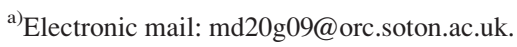

PSBG, the large refractive index contrast between taper and air will require only dozens of periods to achieve a strong grating. Although it is theoretically possible to carve these high contrast gratings in optical fibers, this process would require an extremely long time because of the large amounts of material to be removed. In addition, if a fiber with a high refractive index core $^{8}$ was used to make the taper, the number of grating periods can be decreased even further: by using the large refractive index contrast at the interface between the taper material and air, gratings can be manufactured even with only few periods.

Fig. 1 shows a schematic of the proposed microfiber PSBG resonator with 20 periods $(\Lambda)$ and a $1.5 \Lambda$ phase shift in the center. The cavity is made by inducing a phase shift in the center of a grating inscribed in a microfiber (the uniform waist region of an optical fiber taper). The two conical transition regions at the microfiber extremities connect the cavity to conventional optical fiber pigtails, which allow for a prompt connection to fiberized sources/devices.

The cavity fabrication process involved four main steps: manufacture of optical microfibers; deposition of a thin gold layer; focused ion beam (FIB) nanopatterning and gold etching.

The microfiber fabrication involved heating and stretching a conventional telecom single mode fiber (SMF-1300/ 1550-9/125-0.25-L (OZ optics, Canada) which has $\sim 8.2 \mu \mathrm{m}$ core diameter, $\sim 125 \mu$ m cladding diameter, $\sim 0.12$ numerical aperture and $1250 \mathrm{~nm}$ cut-off wavelength) down to $\sim 2 \mu \mathrm{m}$ using the microheater bushing technique. ${ }^{8}$ To avoid additional optical losses, the microfiber was packaged on a microscope slide coated by a layer of Efiron PC-373 (Luvantix, Ansan-si, Korea), having a cured refractive index of $n$ $\sim 1.373$ at $\lambda=1550 \mathrm{~nm}$. Since bare microfibers undergo rapid mechanical and optical degradation ${ }^{9}$ when their

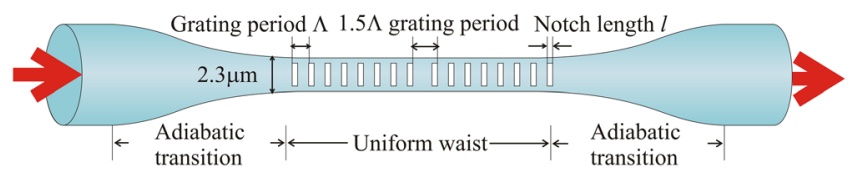

FIG. 1. (Color online) Schematic of the microfiber PSBG. The cavity is created by a phase shift in the center of a grating inscribed in the uniform waist region of an optical fiber taper. 


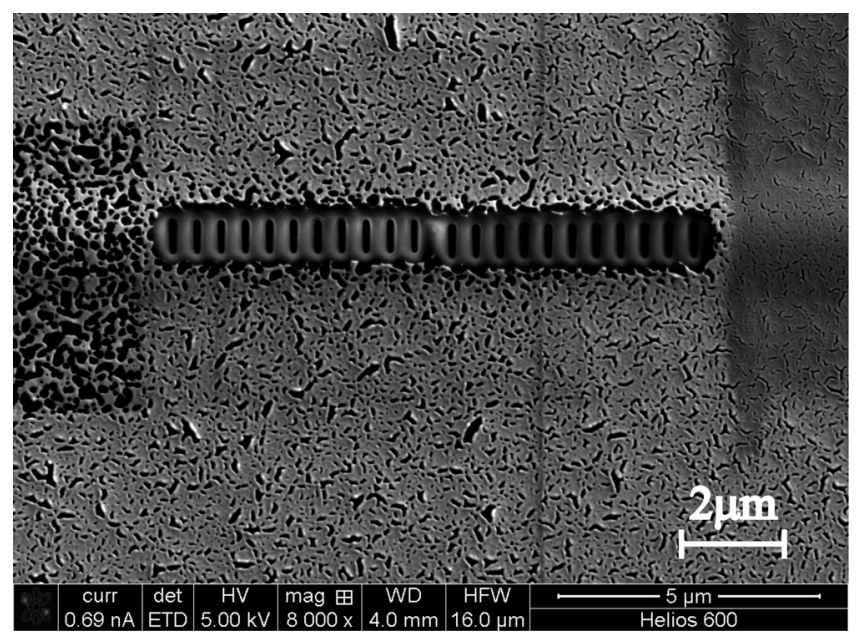

FIG. 2. SEM image of the PSBG. The cavity is at the grating center. The microfiber is partly embedded in a polymer and coated by a $\sim 50 \mathrm{~nm}$ gold layer to avoid charging.

diameter is comparable with the wavelength of the light they guide, to increase the device sturdiness ${ }^{10,11}$ part of the microfiber was embedded in a low refractive index polymer and the microfiber pigtails were fixed to the microscope slide using two small drops of UV curable polymer (UV375, still from Luvantix). A $50 \mathrm{~nm}$ layer of gold was then deposited on the taper surface to avoid charging during FIB milling using an electron beam evaporator. Notches were carved using the FIB system "Helios 600" (FEI Inc., Hillsboro, USA); the Gallium ion beam accelerating voltage and current were $30.0 \mathrm{kV}$ and $93 \mathrm{pA}$, respectively. FIB beam sizes smaller than $30 \mathrm{~nm}$ can be easily obtained, thus notch sizes can be controlled with a good degree of precision. Fig. 2 shows a scanning electron microscope (SEM) image of the gold-coated microfiber PSBG: the notch dimensions, taper diameter and grating periods are $156.2 \mathrm{~nm} \times 718.7 \mathrm{~nm}, \sim 2.3$ $\mu \mathrm{m}$ and $467 \mathrm{~nm}$, respectively; the microcavity length is $687.5 \mathrm{~nm}$. Gold coating was removed after FIB milling was completed.

The device optical properties were characterized with the experimental set-up shown in Fig. 3(a). A supercontinuum (SC) source (Fianium Ltd, U.K.) delivered $50 \mathrm{~nJ}$ pulses over a rather broad range wavelength light (450-1800 nm). The SC source fiberized output was angle cleaved to avoid back reflections. Light from the SC source was launched into the modal filter which eliminated high order modes. ${ }^{12}$ The modal filter was then spliced to a bi-conical $2 \times 2$ microfiber coupler with an extremely wide single mode operation bandwidth (400 nm-1700 nm). ${ }^{13}$ This coupler is specifically designed to suppress any high order mode content present at the input fiber while at the same time providing efficient power splitting into the fundamental mode equally at the two output ports. One of the output ports was connected with the PSBG. Light reflected by the grating passed through the coupler and was recorded by an optical spectrum analyser (OSA) (AQ6317, Yokogawa, Japan). XYZ stages were used to align the different fiber components.

The spectral response of the coupler output was firstly recorded without any sample to provide a normalization base for the following spectra and to remove any wavelength de-

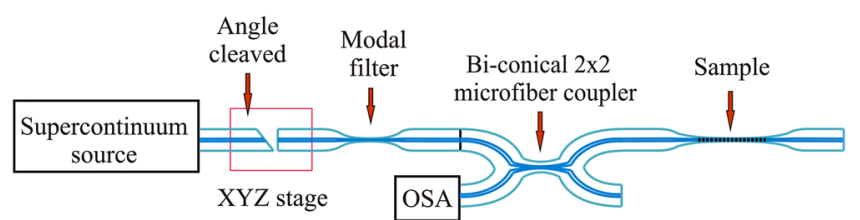

(a)

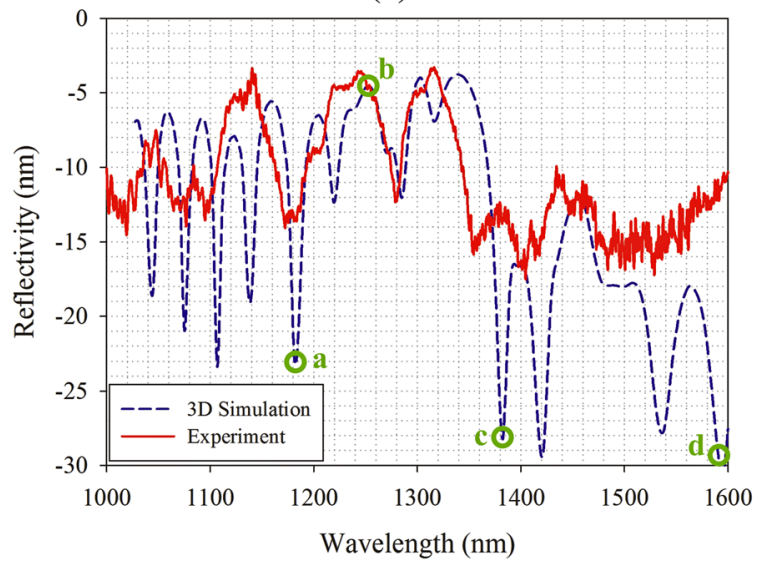

(b)

FIG. 3. (Color online) (a) Schematic of experimental set-up to characterize the PSBG; (b) Reflection spectra of PSBG. The red solid line is experiment result and the blue dash line is simulation line with the same structure as the sample. The points $\mathrm{a}, \mathrm{b}, \mathrm{c}$, and $\mathrm{d}$ in the simulation line represent the wavelengths whose electric fields are showed in Figs. 4(b)-4(e).

pendence related to the source and to the modal filter. Then, the sample reflection spectra were recorded.

Fig. 3(b) shows the reflection response of the proposed microfiber PSBG. The red solid line shows the experiment results while the blue dashed line presents the theoretical prediction data modelled with the dimensions obtained from the SEM image. Experiments show a resonance dip at $\lambda \sim$ $1180 \mathrm{~nm}$, which corresponds to the cavity resonant wavelength. Other dips have been observed at shorter and longer wavelengths are associated with the Fabry-Perot nature of the gratings at either side of the cavity. Yet, these dips are not associated with energy confinement in the cavity region. From the width $(\Delta \lambda)$ and the central position $\left(\lambda_{\mathrm{B}}\right)$ of the
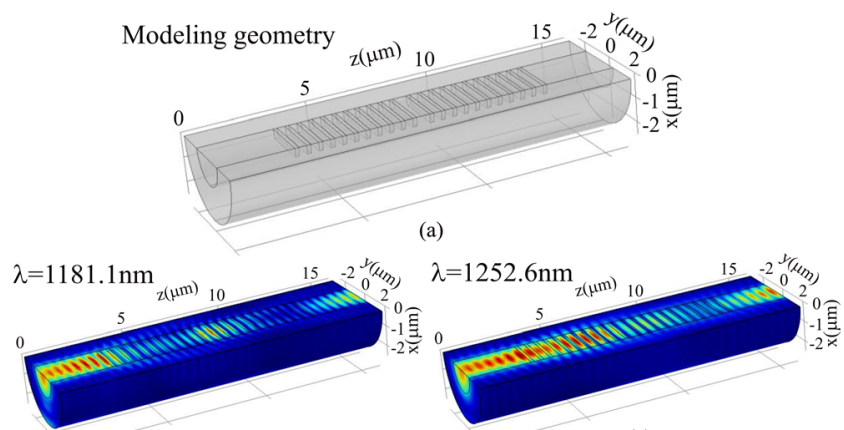

(b)

(d)

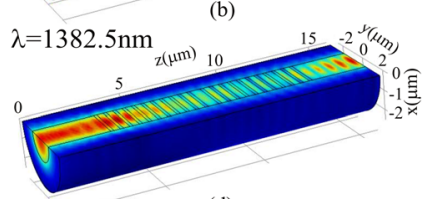

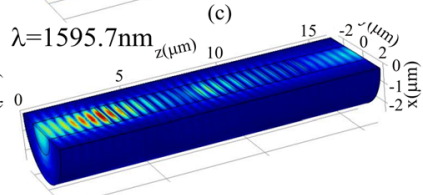

(e)
FIG. 4. (Color online) (a) The modeling geometry; Electric field of simulated PSBG at (b) $\lambda=1181.1 \mathrm{~nm}$, (c) $\lambda=1252.6 \mathrm{~nm}$, (d) $\lambda=1382.5 \mathrm{~nm}$, and (e) $\lambda=1595.7 \mathrm{~nm}$. 


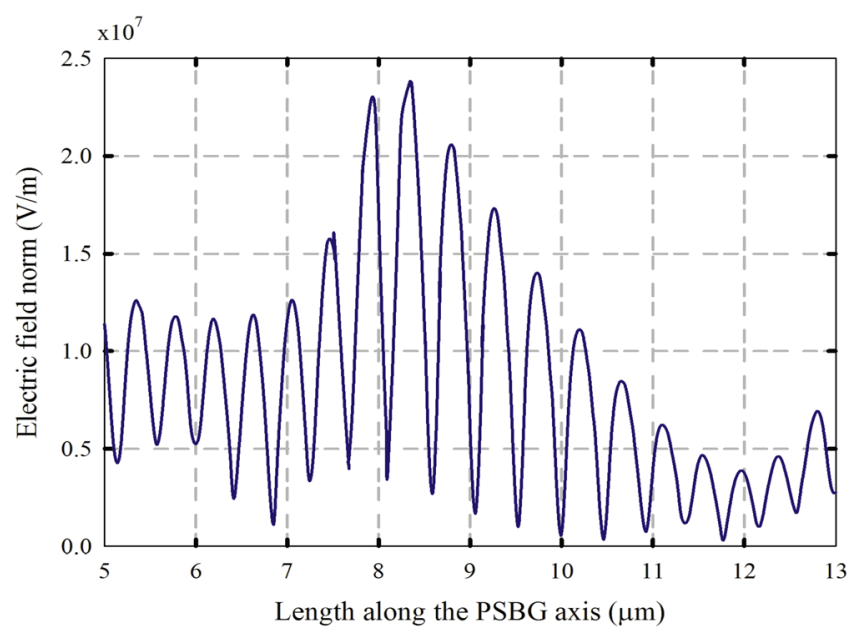

FIG. 5. (Color online) Electric field magnitude against the length along the PSBG axis at $\lambda=1181.1 \mathrm{~nm}$.

cavity dip in Fig. 3(b), it is possible to evaluate the cavity quality factor $(\mathrm{Q})$, defined as: ${ }^{14}$

$$
\mathrm{Q}=\Delta \lambda / \lambda_{\mathrm{B}}
$$

For the manufactured cavity $\mathrm{Q} \sim 60$.

Simulations were carried out to evaluate the microcavity confinement properties using commercial software (COMSOL MULTIPHYSICS 4.1, Stockholm, Sweden). The microfiber diameter $(d)$, grating pitch $(\Lambda)$, central phase shift $(L=1.5 \Lambda)$, notch length $(l)$, and notch width $(w)$ were chosen with the same geometry as the experimental sample. Fig. 4 shows (a) the modeling geometry and (b-e) the electric fields of this structure at four different wavelengths. Due to the symmetry of the structure, a half cylinder was simulated in this study. The chosen boundary conditions were perfect electric conductor at the symmetry plane (flat surface at the top in Fig. 4(a)) and scattering boundary condition at the cylinder outer surfaces. The maximum mesh element sizes were $150 \mathrm{~nm}$ in silica, $300 \mathrm{~nm}$ in polymer and $75 \mathrm{~nm}$ in air. A single mode was launched from the input port. The reflection spectrum was evaluated using the S-parameter function. The reflection spectrum is reported in Fig. 3(b) for comparison with the experimental results, showing a reasonable agreement. The small wavelength peak shift ( $\sim 5 \mathrm{~nm}$ ) shown in Fig. 3(b), can be associated to the surrounding refractive index, which in the simulations is taken as polymer, while in the experiments is a combination of air and polymer (the microfiber is only partially embedded in the polymer). Possible explanations for the other spectral mismatches include the presence of unwanted high order modes, the only partial cavity embedding in the experiments, a different effective index of the mode propagating in the microfiber between experiments and simulations and an imperfect periodicity in the grating notches.
The electric fields were evaluated at different wavelengths and the points a, b, c, and d in Fig. 3(b) represent the wavelengths whose electric fields are showed in Figs. 4(b)4(e): at $\lambda=1181.1 \mathrm{~nm}$ (Fig. 4(b)), light is longitudinal confined in the cavity PSBG center; at $\lambda=1252.6 \mathrm{~nm}$ (Fig. $4(c))$, the grating band-stop wavelength, most of the light is reflected similarly to the case of a simple fiber Bragg grating; electric field at $\lambda=1382.5 \mathrm{~nm}$ (Fig. 4(d)), which is not the resonance dip, shows no confinement; for wavelengths outside the band-stop (Fig. 4(e)), almost all the light can be transmitted through the waveguide. Fig. 5 presents the electric field amplitude along the PSBG axis at $\lambda=1181.1 \mathrm{~nm}$, showing that light was longitudinally confined within $2 \mu \mathrm{m}$ from the center.

Although in this letter only a simple structure is considered, further optimization of manufacturing parameters (such as the grating pitch number and period, the notch width and the length and the microfiber diameter) can provide a more compact device with improved performance. In analogy to the case of phase-shifted fiber Bragg gratings, ${ }^{15}$ the cavity response is related to the grating length and Q-factors in excess of $10^{3}$ can be easily predicted for a larger number of notches.

In conclusion, 3D light confinement was demonstrated in a microfiber PSBG. COMSOL simulations were used to verify that light can be limited in a small volume in the designed structure. This microfiber cavity can be used for a wide range of applications, ranging from sensing to triggered single-photon sources ${ }^{16}$ and the measurement of Casmir effect.

${ }^{1}$ K. Vahala, Nature 424, 839 (2003).

${ }^{2}$ D. K. Armani, T. J. Kippenberg, S. M. Spillane, and K. J. Vahala, Nature 421, 925 (2003).

${ }^{3}$ M. L. Gorodetsky, A. A. Savchenkov, and V. S. Ilchenko, Opt. Lett. 21, 453 (1996).

${ }^{4}$ B. Gayral, J. M. Gerard, A. Lemaitre, C. Dupuis, L. Manin, and J. L. Pelouard, Appl. Phys. Lett. 75, 1908 (1999).

${ }^{5}$ O. Painter, R. K. Lee, A. Scherer, A. Yariv, J. D. O’Brien, P. D. Dapkus, and I. Kim, Science 284, 1819 (1999).

${ }^{6}$ J. M. Gerard, D. Barrier, J. Y. Marzin, R. Kuszelewicz, L. Manin, E. Costard, V. Thierry-Mieg, and T. Rivera, Appl. Phys. Lett. 69, 449 (1996).

${ }^{7}$ G. P. Agrawal and S. Radic, Photon. Technol. Lett. 6, 995 (1994).

${ }^{8}$ G. Brambilla, E. Koizumi, X. Feng, and D. J. Richardson, Electron. Lett. 41, 400 (2005)

${ }^{9}$ G. Brambilla, F. Xu, and X. Feng, Electron. Lett. 42, 517 (2006).

${ }^{10}$ N. Lou, R. Jha, J. L. Domínguez-Juárez, V. Finazzi, J. Villatoro, G. Badenes, and V. Pruneri, Opt. Lett. 35, 571 (2010).

${ }^{11}$ F. Xu and G. Brambilla, Jpn. J. Appl. Phys. 47, 6675 (2008).

${ }^{12}$ Y. Jung, G. Brambilla, and D. J. Richardson, Opt. Express 16, 14661 (2008).

${ }^{13}$ Y. Jung, G. Brambilla, and D. J. Richardson, Opt. Express 17, 5273, (2009).

${ }^{14}$ J. Wilson and J. Hawkes, Optoelectronics: An Introduction (Prentice Hall PTR, Essex, UK, 1998).

${ }^{15}$ R. Zengerle and O. Leminger, J. Lightwave Technol. 13, 2354 (1995).

${ }^{16}$ J. M. Gérard, B. Sermage, B. Gayral, B. Legrand, E. Costard, and V. Thierry-Mieg, Phys. Rev. Lett. 81, 1110 (1998) 